\title{
Frailty and sarcopenia impact surgical and oncologic outcomes after radical cystectomy in patients with bladder cancer
}

\author{
Ram Anil Pathak, Ashok K. Hemal \\ Department of Urology, Wake Forest University School of Medicine, Wake Forest University, Health Sciences Medical Center, Boulevard, Winston- \\ Salem, NC, USA \\ Correspondence to: Ram Anil Pathak; Ashok K. Hemal. Department of Urology, Wake Forest University School of Medicine, Wake Forest University, \\ Health Sciences Medical Center, Boulevard, Winston-Salem, NC 27157-1094, USA. Email: rpathak@wakehealth.edu; ahemal@wakehealth.edu. \\ Provenance: This is an invited Editorial commissioned by Section Editor Xiao Li (Department of Urology, Jiangsu Cancer Hospital \& Jiangsu \\ Institute of Cancer Research \& Nanjing Medical University Affiliated Cancer Hospital, Nanjing, China). \\ Comment on: Mayr R, Fritsche HM, Zeman F, et al. Sarcopenia predicts 90-day mortality and postoperative complications after radical cystectomy for \\ bladder cancer. World J Urol 2018;36:1201-7.
}

Submitted Jul 06, 2018. Accepted for publication Aug 07, 2018.

doi: $10.21037 /$ tau.2018.08.06

View this article at: http://dx.doi.org/10.21037/tau.2018.08.06

Radical cystectomy and urinary diversion are the treatment of choice for muscle-invasive and recurrent, high-grade bladder cancer. Patient outcomes are based on surgical technique (1), the nature/biology of the disease, and patient-specific factors, such as age and co-morbidity status. When assessing the early and long-term complications of cystectomy and urinary diversion (2), it is not only imperative to investigate the biology of the cancer, i.e., grade/stage, presence of hydronephrosis, etc., but also decipher patient-related factors such as frailty and, even more concrete, sarcopenia.

Given the intimate relationship of oncologic and perioperative outcomes with sarcopenia, frailty has been studied extensively in the field of urology. In a series of 137 patients with stage III and IV renal cell carcinoma cancer who underwent radical nephrectomy, the presence of sarcopenia was associated with significantly more perioperative complications (Clavien grade III or higher, $\mathrm{P}=0.03$ ) and poorer oncologic outcomes (node-positive disease, $\mathrm{P}=0.01$ ) (3). With respect to bladder cancer, the impact of sarcopenia on cancer-specific and all-cause mortality has been proven. In a particular robust study with long-term follow-up (6.7 years), sarcopenic patients had significantly poorer cancer-specific survival and overall survival, and sarcopenia was independently found to be linked to all-cause mortality (4).

The authors do a commendable job of re-affirming the notion of the link between sarcopenia and postoperative complications and 90-day mortality in patients undergoing radical cystectomy for urothelial carcinoma of the bladder. In a series of 327 patients, Mayr et al. calculated sarcopenia radiographically using the cross-sectional skeletal muscle surface area at the L3 vertebral level. The authors evaluated 90 -day mortality and postoperative complications and found that sarcopenia, ASA status $(3,4)$, and metastatic disease were significant predictors of 90-day mortality on multi-variable analysis. Furthermore, sarcopenic patients were more likely to suffer major complications (Clavien-Dindo >3b) (2).

The authors suggest that patients with sarcopenia should undergo preoperative counseling given the increased likelihood of 90-day mortality and postoperative complications and offer alternative routes including an adoption of minimally-invasive approaches or a total abandonment of diversion using bowel segments. Although these recommendations might ameliorate some of these complications, the bigger picture of frailty and the frailty index (5) is far more significant than a refinement of surgical technique.

Moreover, the patient population described by the authors may not truly represent the patient population of today. The rate of neoadjuvant chemotherapy, as the authors correctly state, was significantly low, as only $2.4 \%$ of patients received neoadjuvant therapy. Even after 
the knowledge of a known survival benefit, only $8 \%$ of the population from 2012 to 2014 received neoadjuvant chemotherapy.

Unfortunately, sarcopenic patients receiving adjuvant therapy may not benefit similarly than non-sarcopenic patients. In a series of 56 patients undergoing radical cystectomy and adjuvant therapy, a significant difference of approximately 25 months with respect to overall survival was found (6). Perhaps, the best strategy for these patients is aggressive preoperative nutritional and physical support via a multidisciplinary approach, using enhanced recovery after surgery (ERAS) protocol including the consideration of chemotherapy in the neoadjuvant setting, and tailoring surgical techniques [minimally-invasive surgery (MIS) in favor of open surgery] and considering cutaneous ureterostomy to lessen operating time and avoid bowel complications.

\section{Acknowledgements}

None.

\section{Footnote}

Conflicts of Interest: The authors have no conflicts of interest to declare.

Cite this article as: Pathak RA, Hemal AK. Frailty and sarcopenia impact surgical and oncologic outcomes after radical cystectomy in patients with bladder cancer. Transl Androl Urol 2018;7(Suppl 6):S763-S764. doi: 10.21037/tau.2018.08.06

\section{References}

1. Pathak RA, Hemal AK. Editorial Comment on: Rates and Predictors of Conversion to Open Surgery During Minimally Invasive Radical Cystectomy by Ko et al. J Endourol 2018;32:495.

2. Mayr R, Fritsche HM, Zeman F, et al. Sarcopenia predicts 90-day mortality and postoperative complications after radical cystectomy for bladder cancer. World J Urol 2018;36:1201-7.

3. Peyton CC, Heavner MG, Rague JT, et al. Does Sarcopenia Impact Complications and Overall Survival in Patients Undergoing Radical Nephrectomy for Stage III and IV Kidney Cancer? J Endourol 2016;30:229-36.

4. Psutka SP, Carrasco A, Schmit GD, et al. Sarcopenia in patients with bladder cancer undergoing radical cystectomy: impact on cancer-specific and all-cause mortality. Cancer 2014;120:2910-8.

5. Fried LP, Tangen CM, Walston J, et al. Frailty in older adults: evidence for a phenotype. J Gerontol A Biol Sci Med Sci 2001;56:M146-56.

6. Peak T, Colaco M, Hemal A. Does Sarcopenia impact oncologic outcomes in patients undergoing adjuvant chemotherapy following radical cystectomy? J Urol 2017;197:e669-70. 